\title{
Dust-acoustic waves in collisional dusty plasmas of planetary rings
}

\author{
V. V. Yaroshenko ${ }^{1,2}$, F. Verheest ${ }^{3,4}$, and G. E. Morfill ${ }^{1}$ \\ Max-Planck-Institut für extraterrestrische Physik, Giessenbachstrasse, Postfach 1312, 85741 Garching, Germany \\ 2 Institute of Radio Astronomy of National Academy of Sciences of Ukraine, Chervonopraporna 4, 61002 Kharkov, Ukraine \\ Sterrenkundig Observatorium, Universiteit Gent, Krijgslaan 281, 9000 Gent, Belgium \\ ${ }^{4}$ School of Physics, University of KwaZulu-Natal, Private Bag X54001, Durban 4000, South Africa
}

Received 25 July 2006 / Accepted 22 September 2006

ABSTRACT

\begin{abstract}
Aims. The effects of ion and neutral drag forces on stability of low-frequency wave modes is investigated from the point of view of parameters relevant to the plasma environment in the vicinity of the main Saturn rings. The electrons and ions are considered to be magnetized and corotate with the planet, while the dust grains are not magnetized and move around the planet on Keplerian orbits. Methods. The wave modes of low-frequencies in such dusty plasma, propagating along the ring in the azimuthal direction are analyzed based on the susceptibilities derived from the standard fluid approach and model presentation of the drag forces valid in the parameter regime of the dusty plasma of planetary rings.

Results. It is found that the ion drag force can crucially change the stability of dust-acoustic perturbations. Inside the co-rotation distance the ion drag force can be responsible for the excitation of dust-acoustic waves, while for the region outside the synchronous orbit this mode can hardy be excited (at radial distances corresponding to the main rings). The instability due to the ion drag force could be also of some importance for perturbations in remote rings, where the relative ion-dust velocities exceed their thermal speed.
\end{abstract}

Key words. planets: rings - plasmas - waves - instabilities

\section{Introduction}

Complex (dusty) plasmas are characterized by the presence of massive dust grains (with, say, $m_{\mathrm{d}} \simeq 10^{10} m_{\mathrm{p}}-10^{12} m_{\mathrm{p}}$, where $m_{\mathrm{p}}$ is the proton mass) that carry relatively large negative charges (e.g. $Z_{\mathrm{d}} \simeq 10^{2}-10^{3}$ ) in addition to positive ions and electrons. The introduction of new timescales associated with the motion of the slower dust component leads to the existence of an extremely low-frequency dust-wave mode. This is usually referred to as the dust-acoustic wave (DAW) because of the acoustic-like dispersion relation in the long-wavelength limit. The first theoretical consideration of these wave perturbations in isotropic collisionless, weakly coupled complex plasmas was given by Rao et al. (1990).

Complex or dusty plasmas are found in space and astrophysical environments, e.g. in the mesosphere, Saturn's rings, cometary regions and interstellar clouds (Mendis 2005). A variety of laboratory experiments have been carried out on complex plasmas, e.g. studies of collective effects such as waves and instabilities (Chu et al. 1994; D'Angelo 1995; Barkan et al. 1995; Pieper \& Goree 1996; Thompson et al. 1997; Fortov et al. 2000). These experiments confirmed the existence of the DAW mode and suggested useful approaches to complex plasma diagnostics. On the other hand, laboratory studies revealed the important role of collisional processes in the DAW propagation and wave instabilities. Typical peculiarities of waves in laboratory complex plasmas, which should be taken into account when analyzing most experiments, are the following: (i) laboratory complex plasmas are usually weakly ionized and thus strongly collisional, and ion-neutral, ion-dust and dust-neutral collisions become essential factors modifying dust modes; (ii) the plasma electrons and ions stream through the more inertial microparticles due to the discharge electric fields, increasing the role of the ion drag force and leading to various kind of instabilities. These aspects of complex plasmas are studied by Pieper \& Goree (1996), D’Angelo \& Merlino (1996), Rosenberg (1996, 2002), Ivlev et al. (1999), Ivlev \& Morfill (2000), Joice et al. (2002), Khrapak \& Yaroshenko (2003), Khrapak et al. (2003), Fortov et al. (2003) and Yaroshenko et al. (2004).

In space environments, attention has been focused mostly on different kinds of streaming instabilities of low-frequency waves, resulting from relative motion of the plasma components beginning with the work of Bliokh \& Yaroshenko (1985), which dealt with dust plasma waves in Saturn's rings. The excitation of dust-acoustic modes by ions and electrons streaming through the dust grains has been analyzed theoretically by Rosenberg (1993). Melandsø et al. (1993) have given a kinetic model for the dust-acoustic waves applied to planetary rings. They included Landau damping and charge fluctuations due to the collective modes in addition to the velocity difference between dust and plasma. Later, Winske et al. (1995), gave an extensive numerical treatment of the DAW instability. It has been found that the instability saturates by trapping some ions. It was argued that this process could contribute to ion heating and diffusion observed in the inner magnetosphere of Saturn. More information concerning various aspects of collective mode and instabilities in space dusty plasmas can be found in books (Bliokh et al. 1995; Verheest 2000); and some recent contributions (e.g. Li \& Havnes 2000; Cramer et al. 2004). At the same time, space dusty plasmas exhibit many features in common with laboratory complex plasmas. For example, let us consider the dusty plasma in planetary rings. Planetary rings as those of Saturn are made 
up of dust particles imbedded in a plasma, in a plane perpendicular to the rotation axis. Measurements made by the Cassini Plasma Spectrometer Instrument over the main (A and B) rings confirmed the existence of an oxygen atmosphere and ionosphere/plasma in the direct vicinity of Saturn's rings (Waite et al. 2005; Young et al. 2005). The plasma is predicted to be weakly ionized. In particular, Ip (1995) and more recently Waite et al. (2005), gave the ratio of the ion $\mathrm{O}_{2}^{+}$density to the neutral molecular oxygen density $\mathrm{O}_{2}$ as $10^{-3}-10^{-4}$. One can then expect that in a plasma with such low ionization fraction, the collisions of charged particles (electrons, ions and grains) with neutrals cannot be ignored. Furthermore, the dust particles exhibit an azimuthal drift relative to the plasma. The relative velocities varies in a wide range from zero at the region close to synchronous orbit to significant values of the order of the ion thermal velocities at the boundary of the main rings. Such fast relative motion and existence of the ring atmosphere/ionosphere in the vicinity of the rings create the necessary prerequisites for the ion drag force to become a significant factor affecting low-frequency collective modes in the dusty plasma of the planetary rings. Therefore it is necessary to investigate the role of dissipative processes in dust-plasma waves developing on timescales comparable with collisional time scales in this environment.

This paper deals with the effect of ion-neutral, ion-dust and dust-neutral collisions on low-frequency dust-acoustic waves in complex plasmas, from the point of view of parameters relevant to the plasma environment in the vicinity of the Saturn rings. For simplicity we consider only waves propagating along the ring, in the azimuthal direction. Moreover, our model implies that only electrons and ions are subjected to the planetary magnetic field, in the sense that they are bound to the magnetic field in the ring plane and thus co-rotate with the planet as a rigid body. The dust grains are not affected by the magnetic field (due to the small charge-to-mass ratios) and as a result, the grains move on nearly Keplerian orbits with Keplerian frequencies. Finally, we do not take into account the openness of the dusty plasma model, and thus do not consider the processes akin to the ionization instability predicted for laboratory complex plasmas (see e.g. Ivlev et al. 1999; Ivlev \& Morfill 2000).

The plan of the paper as follows. In Sect. 2, we discuss the theoretical formulation of the problem and plasma parameters in the vicinity of the Saturn rings, while in Sect. 3 a linear dispersion relation is derived and some limiting cases are analyzed. The stability analysis is also performed. The obtained results are discussed from the point of view of recent measurements of the plasma parameters performed by the Cassini orbiter (Sect. 4).

\section{General formalism}

\subsection{Basic equations}

In this study of electrostatic dust plasma waves, we deal with a four-component collisional dusty plasma, consisting of plasma electrons (subscript e), ions (subscript i), neutrals (subscript $\mathrm{n}$ ) and negatively charged monodisperse microparticles (subscript d). We restrict our consideration to waves propagating along the ring in the azimuthal direction (the $x$ axis). Furthermore, we will assume that the wave period is much larger than the time scales for dust charge fluctuations to occur, so that the dust charges can be treated as effectively constant.

The plasma species obey the standard set of fluid equations

$$
\frac{\partial v_{\mathrm{e}}}{\partial t}+v_{\mathrm{e}} \frac{\partial v_{\mathrm{e}}}{\partial x}+\frac{v_{T \mathrm{e}}^{2}}{n_{\mathrm{e}}} \frac{\partial n_{\mathrm{e}}}{\partial x}+v_{\mathrm{en}} v_{\mathrm{e}}=-\frac{e}{m_{\mathrm{e}}} E,
$$

$$
\begin{aligned}
& \frac{\partial v_{\mathrm{i}}}{\partial t}+v_{\mathrm{i}} \frac{\partial v_{\mathrm{i}}}{\partial x}+\frac{v_{T \mathrm{i}}^{2}}{n_{\mathrm{i}}} \frac{\partial n_{\mathrm{i}}}{\partial x}+v_{\mathrm{in}} v_{\mathrm{i}}+v_{\mathrm{id}}\left(v_{\mathrm{i}}-v_{\mathrm{d}}\right)=\frac{e}{m_{\mathrm{i}}} E, \\
& \frac{\partial v_{\mathrm{d}}}{\partial t}+v_{\mathrm{d}} \frac{\partial v_{\mathrm{d}}}{\partial x}+v_{\mathrm{dn}} v_{\mathrm{d}}+v_{\mathrm{di}}\left(v_{\mathrm{d}}-v_{\mathrm{i}}\right)=-\frac{Z_{\mathrm{d}} e}{m_{\mathrm{d}}} E, \\
& \frac{\partial n_{\alpha}}{\partial t}+\frac{\partial}{\partial x}\left(v_{\alpha} n_{\alpha}\right)=0 .
\end{aligned}
$$

Here $v_{\alpha}$ and $n_{\alpha}$ refer to the fluid velocities and densities of the different species ( $\alpha=\mathrm{e}, \mathrm{i}, \mathrm{n}, \mathrm{d})$, having mass $m_{\alpha}$, temperature $T_{\alpha}$ and thermal velocities $v_{T \alpha}=\sqrt{T_{\alpha} / m_{\alpha}}$. In the low-frequency dust regime, the dust temperature and pressure effects will be omitted (i.e. $v_{T \mathrm{~d}} \simeq 0$ ). Furthermore, $E=-\mathrm{d} \psi / \mathrm{d} x$ denotes the electric field, where $\psi$ is the electrostatic potential. Regarding the particle charges, we put $q_{\mathrm{e}}=-q_{\mathrm{i}}=-e$ for the plasma electrons and ions, while for the dust grains, the negative particle charge $q_{\mathrm{d}}=-e Z_{\mathrm{d}}$ results from the electron and ion currents flowing to or from the dust grain. Collisions with the neutral gas occur with collision frequencies $v_{\mathrm{en}}, v_{\mathrm{in}}$, and $v_{\mathrm{dn}}$ for the electrons, ions and dust particles, respectively. The other dissipative terms $v_{\text {id }}\left(v_{\mathrm{i}}-v_{\mathrm{d}}\right)$ and $v_{\mathrm{di}}\left(v_{\mathrm{d}}-v_{\mathrm{i}}\right)$ account for the drag of the ions on the charged dust grains and vice versa, with $v_{\mathrm{id}}$ being the iondust momentum-transfer frequency and $v_{\mathrm{di}}=v_{\mathrm{id}} m_{\mathrm{i}} n_{\mathrm{i}} / m_{\mathrm{d}} n_{\mathrm{d}}$ the dust-ion momentum-transfer frequency.

Finally, the set of equations (1)-(4) is closed by the Poisson equation for the electrostatic potential $\psi$

$\frac{\partial^{2} \psi}{\partial x^{2}}=4 \pi e\left(n_{\mathrm{e}}-n_{\mathrm{i}}+Z_{\mathrm{d}} n_{\mathrm{d}}\right)$

\subsection{Plasma parameters in the vicinity of Saturn's rings}

Keeping in mind the application of our studies to the dusty plasmas of planetary rings, we introduce some specific features relevant to this plasma environment. We have estimated the dust plasma parameters on the basis of the scarce data available from the few space missions flown up to now and might well have to be revised in the future. Our estimations of the plasma parameters are listed in Table 1. Most of these values fit approximately those estimated on the basis of the local observations of the Cassini spacecraft in the vicinity of the main $A$ and $B$ rings and given by Waite et al. 2005; Young et al. 2005; Gurnett et al. 2005, while some other quantities especially related to the dust grains (e.g. dust density, temperature, particle size and charge) correspond to plausible assumptions.

According to Waite et al. (2005) and Young et al. (2005), the Cassini orbiter found a ring ionosphere directly over the main $A$ and $B$ rings. The main constituents of the ring-plane ionosphere are oxygen ions $\mathrm{O}_{2}^{+}$and $\mathrm{O}^{+}$. Hydrogen ions $\mathrm{H}^{+}$were also detected, the ratio of the ion densities $\mathrm{O}^{+}$to $\mathrm{O}_{2}^{+}$is about 0.3 , and the ratio of $\mathrm{H}^{+}$to $\mathrm{O}_{2}^{+}$is about 1 (Waite et al. 2005). A tenuous atmosphere in the vicinity of the Saturnian rings is predicted as a consequence of the sputtering of atoms and molecules from the ring particles, resulting from collisions with energetic ions, with micrometeoroids, or with solar protons (Ip 1995).

The plasma temperature varies with distance from the planet and height above the ring midplane, but is typically within a order of a magnitude of $T_{\mathrm{e}} \sim T_{\mathrm{i}} \sim(1-10) \mathrm{eV}$. This yields the electron thermal velocity $v_{T \mathrm{e}} \sim(0.4-1) \times 10^{8} \mathrm{~cm} \mathrm{~s}^{-1}$ and the ion thermal velocities $v_{T \mathrm{i}} \sim(1-3) \times 10^{6} \mathrm{~cm} \mathrm{~s}^{-1}$ for the $\mathrm{H}^{+}$ions, $v_{T \mathrm{i}} \sim(0.3-1) \times 10^{6} \mathrm{~cm} \mathrm{~s}^{-1}$ for the $\mathrm{O}^{+}$ions and $v_{T \mathrm{i}} \sim(2-7) \times 10^{5} \mathrm{~cm} \mathrm{~s}^{-1}$ for $\mathrm{O}_{2}^{+}$ions. At the same time, the frozen plasma electrons and ions co-rotate with the planetary 
Table 1. Estimations of the basic plasma/dust parameters in the vicinity of Saturn's main rings.

\begin{tabular}{|c|c|}
\hline Parameters & Estimates \\
\hline Electron number density & $n_{\mathrm{e} 0} \simeq 2-45 \mathrm{~cm}^{-3}$ \\
\hline Ion $\left(\mathrm{O}_{2}^{+}\right)$number density & $n_{\mathrm{i} 0} \simeq 1-20 \mathrm{~cm}^{-3}$ \\
\hline Ion $\left(\mathrm{O}^{+}\right)$number density & $n_{\mathrm{i} 0} \simeq 0.3-7 \mathrm{~cm}^{-3}$ \\
\hline Ion $\left(\mathrm{H}^{+}\right)$number density & $n_{\mathrm{i} 0} \simeq 1-20 \mathrm{~cm}^{-3}$ \\
\hline Neutral atom number density & $n_{\mathrm{n}} \simeq 10^{3}-10^{4} \mathrm{~cm}^{-3}$ \\
\hline Electron and ion temperature & $T_{\mathrm{e}} \simeq T_{\mathrm{i}} \simeq 1-10 \mathrm{eV}$ \\
\hline Dust grains and neutral temperature & $T_{\mathrm{d}} \simeq T_{\mathrm{n}} \simeq 0.01 \mathrm{eV}$ \\
\hline Ion $\left(\mathrm{O}_{2}^{+}\right)$thermal velocity & $v_{T \mathrm{i}} \simeq 2 \times 10^{5}-7 \times 10^{5} \mathrm{~cm} \mathrm{~s}^{-1}$ \\
\hline Neutral $\left(\mathrm{O}_{2}\right)$ thermal velocity & $v_{T \mathrm{n}} \simeq 2 \times 10^{4} \mathrm{~cm} \mathrm{~s}^{-1}$ \\
\hline Ion $\left(\mathrm{O}^{+}\right)$thermal velocity & $v_{T \mathrm{i}} \simeq 3 \times 10^{5}-10^{6} \mathrm{~cm} \mathrm{~s}^{-1}$ \\
\hline Neutral (O) thermal velocity & $v_{T \mathrm{n}} \simeq 3 \times 10^{4} \mathrm{~cm} \mathrm{~s}^{-1}$ \\
\hline Ion $\left(\mathrm{H}^{+}\right)$thermal velocity & $v_{T \mathrm{i}} \simeq 10^{6}-3 \times 10^{6} \mathrm{~cm} \mathrm{~s}^{-1}$ \\
\hline Neutral $\left(\mathrm{H}_{2}\right)$ thermal velocity & $v_{T \mathrm{n}} \simeq 7 \times 10^{4} \mathrm{~cm} \mathrm{~s}^{-1}$ \\
\hline Electron thermal velocity & $v_{T \mathrm{e}} \simeq 4 \times 10^{7}-10^{8} \mathrm{~cm} \mathrm{~s}^{-1}$ \\
\hline Ion $\left(\mathrm{O}_{2}^{+}\right)$-plasma frequency & $\omega_{\mathrm{pi}}=2.5 \times 10^{2}-1.2 \times 10^{3} \mathrm{~s}^{-1}$ \\
\hline Ion $\left(\mathrm{O}^{+}\right)$-plasma frequency & $\omega_{\mathrm{pi}}=2 \times 10^{2}-8.5 \times 10^{2} \mathrm{~s}^{-1}$ \\
\hline Ion $\left(\mathrm{H}^{+}\right)$-plasma frequency & $\omega_{\mathrm{pi}}=1.3 \times 10^{3}-6 \times 10^{3} \mathrm{~s}^{-1}$ \\
\hline Ion $\left(\mathrm{O}_{2}^{+}\right)$Debye radius & $\lambda_{\mathrm{Di}} \simeq 2 \times 10^{2}-2.4 \times 10^{3} \mathrm{~cm}$ \\
\hline Ion $\left(\mathrm{O}^{+}\right)$Debye radius & $\lambda_{\mathrm{Di}} \simeq 3 \times 10^{2}-5 \times 10^{3} \mathrm{~cm}$ \\
\hline Ion $\left(\mathrm{H}^{+}\right)$Debye radius & $\lambda_{\mathrm{Di}} \simeq 1.5 \times 10^{2}-4 \times 10^{3} \mathrm{~cm}$ \\
\hline Effective ion Debye radius & $\lambda_{\mathrm{D}} \simeq 10^{2}-2 \times 10^{3} \mathrm{~cm}$ \\
\hline Electron Debye radius & $\lambda_{\mathrm{De}} \simeq 1.1 \times 10^{2}-2 \times 10^{3} \mathrm{~cm}$ \\
\hline Ion and electron co-rotation velocity & $v_{\mathrm{i} 0}=v_{\mathrm{e} 0} \simeq(1-3) \times 10^{6} \mathrm{~cm} \mathrm{~s}^{-1}$ \\
\hline Grain drift velocity & $v_{\mathrm{d} 0} \simeq(1-2.5) \times 10^{6} \mathrm{~cm} \mathrm{~s}^{-1}$ \\
\hline Electron-neutral $\left(\mathrm{O}_{2}\right)$ momentum-transfer frequency & $v_{\mathrm{en}} \simeq 10^{-3}-5 \times 10^{-6} \mathrm{~s}^{-1}$ \\
\hline Electron-neutral $(\mathrm{H})$ momentum-transfer frequency & $v_{\mathrm{en}} \simeq 3 \times 10^{-6}-5 \times 10^{-4} \mathrm{~s}^{-1}$ \\
\hline Ion $\left(\mathrm{O}_{2}^{+}\right)$-neutral momentum-transfer frequency & $v_{\text {in }} \simeq 10^{-7}-5 \times 10^{-5} \mathrm{~s}^{-1}$ \\
\hline Ion $\left(\mathrm{H}^{+}\right)$-neutral momentum-transfer frequency & $v_{\mathrm{in}} \simeq 10^{-5}-10^{-4} \mathrm{~s}^{-1}$ \\
\hline Grain size & $a \simeq 1 \mu \mathrm{m}$ \\
\hline Grain mass & $m_{\mathrm{d}}=4 \times 10^{-12} \mathrm{~g}$ \\
\hline Grain charge & $Z_{\mathrm{d}} \simeq 1-10^{2}$ \\
\hline Thermal scattering parameter & $\beta_{T} \simeq 10^{-10}-10^{-8}$ \\
\hline Grain number density & $n_{\mathrm{d}} \simeq 10^{-3}-10^{-1} \mathrm{~cm}^{-3}$ \\
\hline Havnes parameter & $p_{\mathrm{e}}=Z_{\mathrm{d}} n_{\mathrm{d} 0} / n_{\mathrm{e}} \simeq 10^{-3}-0.2$ \\
\hline Plasma-dust frequency & $\omega_{\mathrm{pd}} \simeq 3 \times 10^{-5}-3 \times 10^{-2} \mathrm{~s}^{-1}$ \\
\hline Dust-acoustic velocity & $u_{\mathrm{da}} \simeq\left(10^{-2}-10\right) \mathrm{cm} \mathrm{s}^{-1}$ \\
\hline Grain-neutral $\left(\mathrm{O}_{2}\right)$ momentum-transfer frequency & $v_{\mathrm{dn}} \simeq 10^{-11}-10^{-10} \mathrm{~s}^{-1}$ \\
\hline Grain-neutral $\left(\mathrm{H}_{2}\right)$ momentum-transfer frequency & $v_{\mathrm{dn}} \simeq 5 \times 10^{-12}-5 \times 10^{-11} \mathrm{~s}^{-1}$ \\
\hline Ion $\left(\mathrm{O}_{2}^{+}\right)$-grain momentum-transfer frequency & $v_{\mathrm{i}} \simeq 10^{-5}-5 \times 10^{-3} \mathrm{~s}^{-1}$ \\
\hline Ion $\left(\mathrm{H}^{+}\right)$-grain momentum-transfer frequency & $v_{\mathrm{i}} \simeq 10^{-4}-5 \times 10^{-2} \mathrm{~s}^{-1}$ \\
\hline
\end{tabular}

rotation at the equilibrium velocities $v_{\mathrm{e} 0, \mathrm{i} 0}=\Omega_{\mathrm{p}} r$, with $\Omega_{\mathrm{p}}$ being the planetary rotation frequency (subscripts 0 imply equilibrium quantities), while the dust particles move around the planet with Keplerian velocity $v_{\mathrm{d} 0}=\sqrt{G M_{\mathrm{p}} / r}(G$ is the gravitational constant, $M_{\mathrm{p}}$ denotes the mass of the planet and $r$ is the radial distance from the planet). The two velocities become equal at the synchronous orbit $r^{*}$, determined by $r^{*}=\sqrt[3]{G M_{\mathrm{p}} / \Omega_{\mathrm{p}}^{2}}$. Note that at distances $r<r^{*}$, the dust grains move faster than the electrons and ions, while for $r>r^{*}$, the plasma overtakes the dust particles. Therefore, the relative velocity $\Delta v=\left|v_{\mathrm{i} 0}-v_{\mathrm{d} 0}\right|$ varies in a wide range from zero at $r=r^{*}$ to significant values of the order of $\Delta v \sim 5 \times 10^{5}-10^{6} \mathrm{~cm} \mathrm{~s}^{-1}$ at the boundary of the main rings (e.g. for $D$ and $C$ rings) and can be even larger for the remote $E, F$ or $G$ rings (Fig. 1). This allows us to suggest that in the region of the main planetary rings $(A-C)$ the relative ion velocity does not exceed the ion thermal velocity, namely $U=\left|v_{\mathrm{i} 0}-v_{\mathrm{d} 0}\right| / v_{T \mathrm{i}} \leq 1$, while in the vicinity of the remote planetary rings $(E, G)$ the appropriate regime would be a suprathermal range $(U>1)$.

If there is a relative motion between the dust and plasma components, there inevitably arises a force related to the

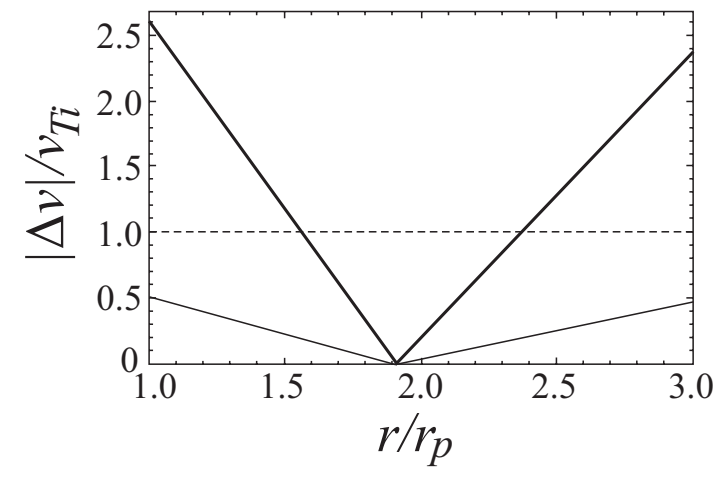

Fig. 1. Relative ion velocity $\left|v_{\mathrm{i} 0}-v_{\mathrm{d} 0}\right| / v_{T \mathrm{i}}$ vs. the normalized distance from the planet $r / r_{\mathrm{p}}$ : bold solid line for oxygen $\left(\mathrm{O}_{2}^{+}\right)$and thin solid line for hydrogen $\left(\mathrm{H}^{+}\right)$ions. The ion temperature is taken as $10 \mathrm{eV}$. The main Saturn rings $C, B$ and $A$ are located at $r / r_{\mathrm{p}} \sim 1.25-2.21$.

exchange of momentum transfer from a flowing plasma to charged dust grains. Because of their much larger masses, ions mainly contribute to this drag force. Recently, there has been 
a considerable theoretical development in modelling of the ion drag force (Barnes et al. 1992; Kilgore et al. 1993; Daugherty $\&$ Graves 1995; Khrapak et al. 2002). The ion drag force $F_{\mathrm{i}}$ consists of two parts often referred to as collection and orbital forces. The collection force is associated with momentum transfer from the ions that are collected by the grain, while the orbital force is due to the momentum transfer from the ions that are scattered in the electric field of the grain. The important role in the modelling of $F_{\mathrm{i}}$ plays the so-called scattering parameter, $\beta$, which is determined by the ratio of the Coulomb radius of ion-grain interactions, $R_{\mathrm{c}}$, to the plasma screening length $\lambda_{D}$, i.e. $\beta_{T}=R_{\mathrm{c}} / \lambda_{D}$. In the vicinity of the main rings, where $U \leq 1$, the use of the thermal scattering parameter $\beta_{T}=Z_{\mathrm{d}} e^{2} / T_{\mathrm{i}} \lambda_{D}$ is more appropriate. For typical values of the Debye length $\lambda_{D} \sim 1-10 \mathrm{~m}$ and assuming particle charges $Z_{\mathrm{d}} \sim 1-100$, one always obtains $\beta_{T} \ll 1$. This limit corresponds to weak coupling between the ions and dust particles. Moreover, it turns out that for typical plasma parameters, the scattering parameter is so small that $\beta_{T} \sim 10^{-10}-10^{-8}<a / \lambda_{D}$ or even $\beta_{T} \ll a / \lambda_{D}$, and the ion drag force is mainly specified by the collection part. The ion-dust momentum-transfer frequency $v_{\text {id }}$ can then be approximated as $v_{\mathrm{id}} \simeq(8 \sqrt{2 \pi} / 3) a^{2} n_{\mathrm{d}} v_{T \mathrm{i}}$ (Barnes et al. 1992; Khrapak et al. 2002) leading to $v_{\mathrm{di}} \simeq(8 \sqrt{2 \pi} / 3) a^{2} n_{\mathrm{i}} m_{\mathrm{i}} v_{T \mathrm{i}} / m_{\mathrm{d}}$ and hence the ion drag force becomes $F_{\mathrm{i}} \simeq v_{\mathrm{di}} v_{\mathrm{i}}$.

For the momentum transfer frequency in dust-neutral collisions we adopt the standard theory (Epstein 1921) yielding $v_{\mathrm{dn}} m_{\mathrm{d}}=(8 \sqrt{2 \pi} / 3) \delta a^{2} n_{\mathrm{n}} m_{\mathrm{n}} v_{T \mathrm{n}}$, where $m_{\mathrm{n}}, n_{\mathrm{n}}$ and $v_{T \mathrm{n}}=\sqrt{T_{\mathrm{n}} / m_{\mathrm{n}}}$ are the mass, density and thermal velocity of neutrals, respectively. According to Liu et al. 2003, the numerical factor $\delta \simeq 1.4$ accounts for diffuse scattering. The ratio $v_{\mathrm{di}} / v_{\mathrm{dn}} \propto n_{\mathrm{i}} v_{T \mathrm{i}} / n_{\mathrm{n}} v_{T \mathrm{n}}$ is then specified by the relative number densities of the ions and neutral components $\left(n_{\mathrm{i}} / n_{\mathrm{n}}\right)$ and the square root of the temperature ratio $\sqrt{T_{\mathrm{i}} / T_{\mathrm{n}}}$. Even if we assume that the neutrals are much colder than the ions (e.g. $T_{\mathrm{n}} \sim 0.01 \mathrm{eV} \ll T_{\mathrm{i}} \geq 1 \mathrm{eV}$ ), $v_{\mathrm{di}} / v_{\mathrm{dn}}$ remains small enough since $n_{\mathrm{i}} \ll n_{\mathrm{n}}$. Nevertheless, we cannot neglect the ion drag force, $F_{\mathrm{i}}$ in the momentum equation for the dust component, since $F_{\mathrm{i}} \simeq v_{\mathrm{di}} v_{\mathrm{i}}$ can be of the same order as a neutral drag force $F_{\mathrm{n}}=v_{\mathrm{dn}} v_{\mathrm{d}}$ due to $v_{\mathrm{i}} \gg v_{\mathrm{d}}$ and the force ratio $F_{\mathrm{i}} / F_{\mathrm{n}} \simeq\left(n_{\mathrm{i}} v_{\mathrm{i}} / n_{\mathrm{n}} v_{\mathrm{d}}\right) \sqrt{T_{\mathrm{i}} / T_{\mathrm{n}}}$ can easily become $\geq 1$ or even much large than 1 .

It is also instructive to compare the ion-neutral and ion-dust momentum-transfer frequencies. In the limit of subthermal ion drift $\left(v_{\mathrm{i} 0} \lesssim V_{T \mathrm{i}}\right)$, ion neutral momentum transfer frequency can be estimated as $v_{\text {in }}=n_{\mathrm{n}} \sigma_{\mathrm{in}} v_{T \mathrm{i}}$, where $n_{\mathrm{n}}$ denotes the neutral number density and $\sigma_{\text {in }}$ is the effective collisional cross section (accounting for both elastic scattering due to polarization interactions with neutrals and charge exchange). For subthermal and slightly superthermal oxygen ions $\sigma_{\text {in }} \sim 10^{-14}-10^{-15} \mathrm{~cm}^{2}$ (Smith et al. 1996), thus yielding $v_{\text {in }} / v_{\text {id }}=\left(10^{-7}-10^{-8}\right) n_{\mathrm{n}} / n_{\mathrm{d}}$. Depending on the particle density, the ratio $v_{\text {in }} / v_{\text {id }}$ leads to domination either of the neutral drag force or of the force associated with ion-dust collisions. The typical solid particle size in some of Saturns rings is about a micron or less. The number density of the micron-sized grains being one of the most uncertain parameters of the problem, we chose $n_{\mathrm{d}} \sim 10^{-3}-10^{-1} \mathrm{~cm}^{-3}$, which is often used for dusty plasmas in planetary rings (Bliokh et al. 1995; Li \& Havnes 2000; Cramer et al. 2004). Therefore, to be realistic, we have to keep both terms $\left(v_{\mathrm{in}}, v_{\mathrm{id}}\right)$ in the ion momentum equation.

Finally note that for the estimations of the electron neutral momentum transfer frequency $v_{\mathrm{en}}=n_{\mathrm{n}} \sigma_{\mathrm{en}} v_{T \mathrm{e}}$ listed in Table 1 we have used the total cross section given by Itikawa et al. (1989), $\sigma_{\mathrm{en}} \sim 4 \times 10^{-16}-10^{-15} \mathrm{~cm}^{2}$ for electron collisions with oxygen atoms and molecules and take $\sigma_{\mathrm{en}} \sim 10^{-16} \mathrm{~cm}^{2}$ for electron collisions with hydrogen atoms.

\section{Dispersion equation}

Following the standard procedure, we linearize the basic Eqs. (1)-(5) with respect to small perturbations $v_{\alpha} \rightarrow v_{\alpha 0}+v_{\alpha}$; and $n_{\alpha} \rightarrow n_{\alpha 0}+n_{\alpha} ; \psi \rightarrow \psi$, where the values with the subscript " 0 " refer to the equilibrium state of the plasma. Note that in equilibrium, the charge neutrality condition gives $n_{\mathrm{i} 0}-n_{\mathrm{e} 0}-$ $Z_{\mathrm{d}} n_{\mathrm{d} 0}=0$. All the first-order quantities are supposed to vary as $\exp (-\mathrm{i} \omega t+\mathrm{i} k x)$. Introducing the susceptibilities for all plasma species as $\chi_{\alpha}=-4 \pi q_{\alpha} n_{\alpha} / k^{2} \psi$, and inserting these in Poisson's equation (5) gives the dispersion law

$1+\chi_{\mathrm{i}}+\chi_{\mathrm{e}}+\chi_{\mathrm{d}}=0$.

Furthermore, we use the common assumption that compared to the heavy ions and dust grains, the plasma electrons can be considered as effectively massless. Then only the Boltzmann distribution

$n_{\mathrm{e}}=n_{\mathrm{e} 0} \exp \left(\frac{e \psi}{T_{\mathrm{e}}}\right)$

remains of Eq. (1) with an equilibrium electron density $n_{\mathrm{e} 0}$. The electron susceptibility then reduces with the help of (7) to

$\chi_{\mathrm{e}}=\frac{1}{k^{2} \lambda_{\mathrm{De}}^{2}}$

with $\lambda_{\mathrm{De}}=\sqrt{T_{\mathrm{e}} / 4 \pi e^{2} n_{\mathrm{e} 0}}$. Note that the obtained electron susceptibility is valid in a low frequency regime $\omega \ll k v_{T e}$, $k^{2} v_{T \mathrm{e}}^{2} / v_{\mathrm{en}}$.

The ion and dust drift influence, on the other hand, will be kept in the main equations. Indeed, elimination of all quantities in favor of the ion density fluctuations $n_{\mathrm{i}}$ from (2)-(5) yields

$\chi_{\mathrm{i}}=-\frac{\omega_{\mathrm{pi}}^{2}\left[\left(\omega-k v_{0 \mathrm{~d}}\right)\left(\omega-k v_{0 \mathrm{~d}}+\mathrm{i} v_{\mathrm{dn}}\right)+\mathrm{i} \delta v_{\mathrm{i}} p k \Delta v\right]}{\left(\omega-k v_{0 \mathrm{~d}}\right)\left[\left(\omega-k v_{0 \mathrm{~d}}+\mathrm{i} v_{\mathrm{dn}}\right) L_{\mathrm{i}}-\delta v_{\mathrm{i}}^{2} k \Delta v\right]}$

Here we have used the notations for the unperturbed values $v_{\mathrm{i}}=v_{0 \mathrm{id}}, \Delta v=\left(v_{\mathrm{i} 0}-v_{\mathrm{d} 0}\right), n_{\mathrm{d} 0} Z_{\mathrm{d}} / n_{\mathrm{i} 0}=p$ (the ion Havnes parameter) and $\delta=m_{\mathrm{i}} n_{\mathrm{i} 0} / m_{\mathrm{d}} n_{\mathrm{d} 0}$. Furthermore we denote $L_{\mathrm{i}}=$ $\left(\omega-k v_{\mathrm{i} 0}\right)\left(\omega-k v_{\mathrm{i} 0}+i\left(v_{\mathrm{i}}+v_{\mathrm{in}}\right)\right)-k^{2} v_{T \mathrm{i}}^{2}$. The plasma frequencies are given by $\omega_{\mathrm{p} \alpha}^{2}=4 \pi q_{\alpha}^{2} n_{\alpha 0} / m_{\alpha}$ with $(\alpha=\mathrm{e}, \mathrm{i}, \mathrm{d})$.

Finally, using (9), the initial set of equations (2)-(5) can then be reduced to the following expression for the dust susceptibility

$\chi_{\mathrm{d}}=-\frac{\omega_{\mathrm{pd}}^{2}\left[L_{\mathrm{i}}-\mathrm{i} v_{\mathrm{i}} p^{-1}\left(\omega-k v_{\mathrm{d} 0}\right)\right]}{\left(\omega-k v_{\mathrm{d} 0}\right)\left[\left(\omega-k v_{\mathrm{d} 0}+\mathrm{i} v_{\mathrm{dn}}\right) L_{\mathrm{i}}-\delta v_{\mathrm{i}}^{2} k \Delta v\right]}$

As can be seen from (8)-(10) the general dispersion relation (6) becomes too involved to be analyzed directly. In view of the properties of dusty plasmas in the vicinity of the planetary ring, we will use the following reasonable approximations:

(i) We will consider perturbations, whose length-scales, $\Lambda$, could be noticeable upon the ring structure. As a minimal scale, the characteristic size of "spokes" along the ring is taken, giving $\Lambda \geq 10^{5}-10^{6} \mathrm{~cm}$, while the maximal length is constrained from above by the condition $\Lambda \ll r^{*} \sim 10^{10} \mathrm{~cm}$, thus leading to the wavenumber range $10^{-5}>k>10^{-8}\left(\mathrm{~cm}^{-1}\right)$. The perturbations can therefore be defined as long-wavelength ones, since always $k \lambda_{\mathrm{De}} \sim k \lambda_{\mathrm{Di}} \ll 1$ 
(ii) For the plasma parameters in the vicinity of the planetary rings (Table 1), in the considered wavelength domain, the term $\delta v_{\mathrm{i}}^{2} k \Delta v$ is always small compared to $k^{3} v_{\mathrm{i} 0}^{2} v_{\mathrm{d} 0}$ or $k^{3} v_{T \mathrm{i}}^{2} v_{\mathrm{d} 0}$, so that we will neglect the second term between square brackets in the denominators of Eqs. (9) and (10).

The dispersion relation is then simplified to

$$
\begin{aligned}
1-\frac{k^{2} u_{\mathrm{ia}}^{2}}{L_{\mathrm{i}}} & -\frac{k^{2} u_{\mathrm{da}}^{2}}{\left(\omega-k v_{\mathrm{d} 0}\right)\left(\omega-k v_{\mathrm{d} 0}+\mathrm{i} v_{\mathrm{dn}}\right)}+\mathrm{i} k^{2} u_{\mathrm{da}}^{2} v_{\mathrm{i}} p^{-1} \\
& \times \frac{\left(\omega-k v_{\mathrm{i} 0}\right)}{\left(\omega-k v_{\mathrm{d} 0}\right)\left(\omega-k v_{\mathrm{d} 0}+\mathrm{i} v_{\mathrm{dn}}\right) L_{\mathrm{i}}}=0
\end{aligned}
$$

with an ion-sound velocity $u_{\mathrm{ia}}=\lambda_{\mathrm{De}} \omega_{\mathrm{pi}} \simeq v_{T \mathrm{i}}$ (as a result of the assumption $T_{\mathrm{e}} \simeq T_{\mathrm{i}}, n_{\mathrm{e} 0} \simeq n_{\mathrm{i} 0}$ ) and a dust-sound velocity $u_{\mathrm{da}}=\lambda_{\mathrm{De}} \omega_{\mathrm{pd}} \simeq \lambda_{\mathrm{Di}} \omega_{\mathrm{pd}}$. The appearance of the last term in the dispersion relation (11) is new, resulting from the coupling between perturbations of the ion and dust particle populations (densities) due to the ion drag force. It should be noted that this contribution (due to the momentum exchange between the dust component and plasma ions) can be positive or negative, depending on the distance from the planet and the relation between $\Delta v$ and $v_{T \mathrm{i}}$. As a result one can expect the existence of spatially restricted domains where the dust-wave be generated.

\section{Dust-acoustic solutions}

Analyzing the dispersion relation, we start with the simplest case of the region close to synchronous orbit $r \sim r^{*}$, where $v_{\mathrm{d} 0} \simeq v_{\mathrm{i} 0}$. We will work in the planetary frame of reference. Putting then $\omega-k v_{\mathrm{i} 0} \simeq \omega-k v_{\mathrm{d} 0} \rightarrow \omega$ in (11) yields

$1-\frac{k^{2} v_{T \mathrm{i}}^{2}}{L_{\mathrm{i}}}-\frac{k^{2} u_{\mathrm{da}}^{2}}{\omega\left(\omega+\mathrm{i} v_{\mathrm{dn}}\right)}+\frac{\mathrm{i} k^{2} u_{\mathrm{da}}^{2} v_{\mathrm{i}}}{\left(\omega+\mathrm{i} v_{\mathrm{dn}}\right) L_{\mathrm{i}} p}=0$,

where the term $L_{\mathrm{i}}$ reduces to $L_{\mathrm{i}}=\omega^{2}+\mathrm{i} \omega\left(v_{\mathrm{i}}+v_{\mathrm{in}}\right)-k^{2} v_{T \mathrm{i}}^{2}$. To study solutions of Eq. (12) in the dust regime, we use the ordering $k v_{T \mathrm{i}} \gg \omega \gg v_{\mathrm{dn}}$, which enables us to linearize the contributions of the last two terms. Equation (12) can then easily be solved for weak growth (or damping), using $\omega=\mathfrak{R} \omega+\mathrm{i} \mathfrak{J} \omega$ with $|\mathfrak{J} \omega| \ll \mathfrak{R} \omega$. This yields in the low-frequency regime

$\mathfrak{R} \omega \simeq k u_{\mathrm{da}} / \sqrt{2}, \quad \mathfrak{J} \omega \simeq \frac{u_{\mathrm{da}}^{2}\left(2 v_{\mathrm{i}}-v_{\mathrm{in}} p\right)}{8 v_{T \mathrm{i}}^{2} p}-\frac{v_{\mathrm{dn}}}{2}$.

To derive these expressions we have used the plausible assumptions $v_{T \mathrm{i}}^{2} \gg u_{\mathrm{da}}^{2}$ and $p \ll 1$. The thus obtained solution describes the usual dust-acoustic mode (the numerical factor $\sqrt{2}$ follows from the use of the ion (electron) Debye length instead of the linearized screening length $\left.\lambda_{\mathrm{D}}=\left(\sqrt{\lambda_{\mathrm{De}}^{-2}+\lambda_{\mathrm{Di}}^{-2}}\right)^{-1} \simeq \lambda_{\mathrm{De}} / \sqrt{2}\right)$. The dust-acoustic solution (13) corresponds to either unstable $(\mathfrak{J} \omega>0)$ or stable (damped, $\mathfrak{J} \omega<0$ ) wave perturbations, depending on the specific values of the plasma parameters. The instability due to the ion drag force requires

$v_{\mathrm{i}}>v_{\mathrm{cr}}=p\left(2 v_{T \mathrm{i}}^{2} v_{\mathrm{dn}} / u_{\mathrm{da}}^{2}+v_{\mathrm{in}} / 2\right) \simeq 2 v_{\mathrm{dn}} p v_{T \mathrm{i}}^{2} / u_{\mathrm{da}}^{2}$,

which can be simplified to $v_{\mathrm{i}}>v_{\mathrm{cr}} \simeq 2 v_{\mathrm{dn}} m_{\mathrm{d}} /\left(m_{\mathrm{i}} Z_{\mathrm{d}}\right)$. Inserting explicit expressions for $v_{\mathrm{dn}}$ and $v_{\mathrm{i}}$, one can reduce the discussion of the wave stability to two variables - the dust charge density fraction (relative to the neutral atoms) $p_{\mathrm{n}}=Z_{\mathrm{d}} n_{\mathrm{d} 0} / n_{\mathrm{n}}$ and the square root of the ratio of the ion and neutral temperatures, $s=\sqrt{T_{\mathrm{i}} / T_{\mathrm{n}}}$. As an illustration, we plot in Fig. 2 the existence domain for growing dust-acoustic waves in the parameter space

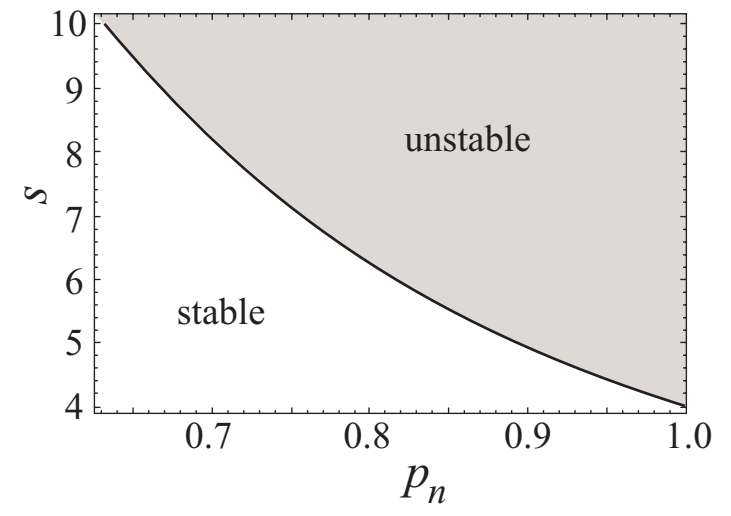

Fig. 2. Instability domain in the parameter space $\left(p_{\mathrm{n}}=Z_{\mathrm{d}} n_{\mathrm{d} 0} / n_{\mathrm{n}} ; s=\right.$ $\left.\sqrt{T_{\mathrm{i}} / T_{\mathrm{n}}}\right)$ calculated for dust grains orbiting in the vicinity of the synchronous orbit $r \sim r^{*}$.

$p_{\mathrm{n}}=Z_{\mathrm{d}} n_{\mathrm{d} 0} / n_{\mathrm{n}}$ and $s=\sqrt{T_{\mathrm{i}} / T_{\mathrm{n}}}$. In the grey region only unstable solutions due to the ion drag force can be found. The damped dust-acoustic perturbations exist outside this region. The range of the instability occurrence $\left(v_{\mathrm{i}}>v_{\mathrm{cr}}\right)$ is limited by rather specific conditions: it requires high values of $s=\sqrt{T_{\mathrm{i}} / T_{\mathrm{n}}}>1$ and $p_{\mathrm{n}} \leq 1$. However, typically $p_{\mathrm{n}}=Z_{\mathrm{d}} n_{\mathrm{d} 0} / n_{\mathrm{n}} \ll 1$ and $s \geq 1$, so that the dust-acoustic instability due to the ion drag force can hardly develop around the synchronous orbit.

To explore the influence of a finite difference in equilibrium velocities on the low-frequency solutions (11), we replace $\omega-$ $k v_{\mathrm{d} 0} \rightarrow \omega$. We first consider the case when $\Delta v=v_{\mathrm{i} 0}-v_{\mathrm{d} 0}<v_{T \mathrm{i}}$ and use the ordering $v_{\mathrm{dn}} \ll \omega \ll k \Delta v$. One then easily finds that the DAW mode drifting along with the dust flow obeys

$\mathfrak{R} \omega \simeq k u_{\mathrm{da}} / \sqrt{2}, \quad \mathfrak{J} \omega \simeq-\frac{\sqrt{2} v_{\mathrm{i}} u_{\mathrm{da}} \Delta v}{4 v_{T \mathrm{i}}^{2} p}-\frac{v_{\mathrm{dn}}}{2}$.

For these perturbations, the instability due to the ion drag force can only occur in that part of the planetary rings where the ions move slower than the dust grains, i.e. inside the co-rotation distance, at orbits $r<r^{*}$, giving $\Delta v<0$. The instability threshold is now "reduced" to $v_{\mathrm{cr}}=\sqrt{2} v_{T \mathrm{i}}^{2} p v_{\mathrm{dn}} /\left(u_{\mathrm{da}} \Delta v\right)$, which is $\left(u_{\mathrm{da}} / \Delta v \ll 1\right)$ times lower than (13), and thus can be easily achieved. It is instructive to rewrite the instability criterion in terms of $\Delta v$ as

$|\Delta v|>\sqrt{2} \frac{v_{\mathrm{dn}} p v_{T \mathrm{i}}^{2}}{v_{\mathrm{i}} u_{\mathrm{da}}}$

Our discussion is thus restricted to values of $|\Delta v|$, the lower limit of which is determined by the right hand side of (16), while the upper one is given by the condition $|\Delta v|<v_{T i}$. In contrast to the case of the dust moving at the synchronous orbit $r \sim r^{*}$, the instability criterion (16) is now dependent on specific particle positions in the ring (see Fig. 3). If the dusty plasma is homogeneous within the ring plane, the instability can only be found for particles orbiting at $r<r_{\mathrm{cr}}$, yielding $v_{\mathrm{i}}>v_{\mathrm{cr}}\left(r_{\mathrm{cr}}\right.$ represents the distance where $|\Delta v|$ becomes equal to the right hand side of (16)).

The nature of the instability considered can be easily understood, by considering the equation of the energy balance. According to the standard way (see e.g. Mikailovskii 1973)

$\frac{\mathrm{d}}{\mathrm{d} t} W_{k}=-E_{0}^{2} \omega_{k} \frac{\mathfrak{J} \varepsilon\left(\omega_{k}, k\right)}{4 \pi}$

where the wave energy is

$W_{k}=\frac{E_{0}^{2}}{8 \pi} \omega_{k} \frac{d}{d \omega_{k}} \mathfrak{R} \varepsilon$ 


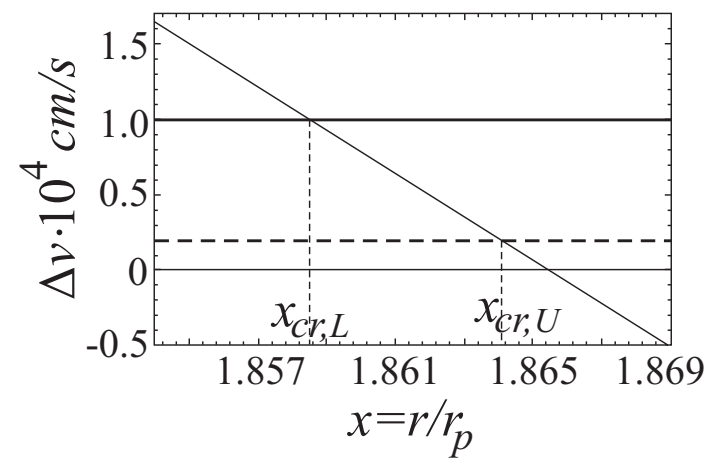

Fig. 3. The instability region in parameter space $\left(x=r / r_{\mathrm{p}},|\Delta v|\right)$ is determined by the condition $r<r_{\text {cr }}$ (with $r_{\text {cr }}$ being the distance where $|\Delta v|$ equals the threshold value given by Eq. (16)). The horizontal lines correspond to the upper and lower limits of the threshold value (16) calculated for typical plasma parameters, listed in Table 1.

Here $E_{0}$ denotes the wave amplitude and the dielectric permeability of the dusty plasma $\varepsilon\left(\omega_{k}, k\right)$ is given by the left-hand side of Eq. (12). The quantity $\omega_{k} \mathfrak{J} \varepsilon\left(\omega_{k}, k\right) / 4 \pi=\sigma$ has the meaning of a plasma conductivity and therefore the right hand side of the balance Eq. (17) $E_{0}^{2} \sigma$ represents the amount of the wave energy dissipated in unit time. Finally, the subscript $k$ specifies a solution of the dispersion relation $\mathfrak{R} \varepsilon\left(\omega_{k}, k\right)=0$.

One easily finds that for the dust-acoustic wave propagating along the dust flow (15), the wave energy and the plasma conductivity are determined as $W_{k} \simeq-\sqrt{2} E_{0}^{2} \Delta v / 2 \pi u_{\mathrm{da}}$ and $\sigma \simeq-\Delta v\left(\frac{v_{\mathrm{i}} \Delta v}{p v_{T \mathrm{i}}^{2}}+\frac{\sqrt{2} v_{\mathrm{dn}}}{u_{\mathrm{da}}}\right) / 2 \pi$. When the difference between the flow velocities $\Delta v$ is positive, $W_{k}<0$ and $\sigma<0$ and therefore, one deals with the damped negative energy DAW mode. Only in the case $\Delta v<0$, the wave energy becomes positive, but the plasma conductivity remains negative for the regime prescribed by (16). The latter means that the picture of the DAW growth corresponds to the dissipative instability resulting from anomalous dissipation (Mikailovskii 1973). The amplitude of the positive energy DAW grows due to the energy obtained from the relative motion between the plasma components via the ion drag force.

A further growth of the relative dust-plasma velocity, $|\Delta v|=$ $\left|v_{\mathrm{i} 0}-v_{\mathrm{d} 0}\right| \sim v_{T \mathrm{i}}$ will result in unstable (damped) modifications of the dust-acoustic perturbations, $\mathfrak{R} \omega \sim|\mathfrak{J} \omega|$, developing with

$\mathfrak{J} \omega \simeq \pm k u_{\mathrm{da}} \frac{\sqrt{v_{\mathrm{i}}}}{\sqrt{2 p k v_{T \mathrm{i}}}}$.

These expressions were derived using the reasonable assumptions that $\mathfrak{R} \omega, \mathfrak{J} \omega>v_{\mathrm{dn}}$ and $k|\Delta v|>v_{\mathrm{in}}, v_{\mathrm{i}}$. The instability (+) due to the ion drag force can only develop for dust orbiting at $r \sim r_{T}<r^{*}$, where $-\Delta v\left(r_{T}\right) \sim v_{T i}$, the reversed position outside the corotation orbit $r \sim 2 r^{*}-r_{T}>r^{*}$ (here the ions move faster than the dust grains and thus provide $\left.\Delta v(r) \sim v_{T i}\right)$ corresponding to the damped mode ("-" sign in Eq. (19)).

Finally, for larger relative dust-plasma velocities, when $\Delta v>v_{T \mathrm{i}}$ or even $\Delta v \gg v_{T \mathrm{i}}$, and $k|\Delta v|>v_{\text {in }}, v_{\mathrm{i}}$, one obtains the approximate dust-acoustic solution of (11) in the form

$\mathfrak{R} \omega \simeq \frac{k u_{\mathrm{da}} \Delta v}{\sqrt{\Delta v^{2}-2 v_{T \mathrm{i}}^{2}}}, \quad \mathfrak{J} \omega \simeq-\frac{v_{\mathrm{dn}}}{2}+\frac{1}{2} \frac{u_{\mathrm{da}} v_{\mathrm{i}}}{p \Delta v}$.

As can be seen, the ion-dust collisions can also trigger the dustacoustic instability in the region $r \gg r^{*}$ (where $\Delta v>0$ ) when, on the one hand, the velocity difference is large enough so that

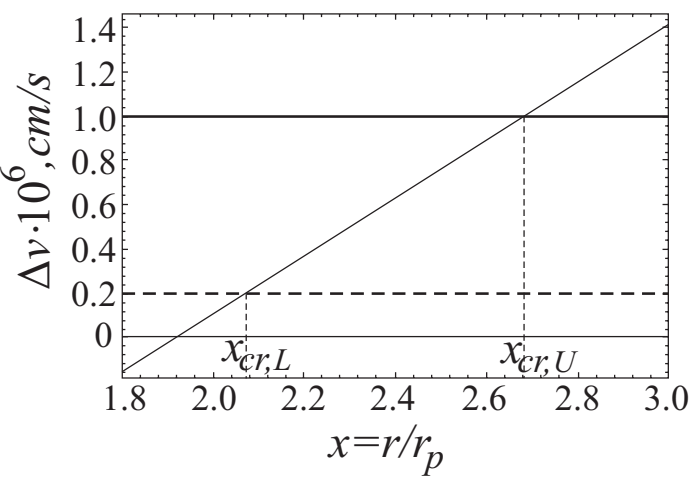

Fig. 4. The instability domain in parameter space $\left(x=r / r_{\mathrm{p}}, \Delta v\right)$ is determined by a condition $r>r_{\mathrm{cr}}=2 r^{*}-2 r_{T}$ (with $r_{T}$ being the distance where $\left.\left|\Delta v\left(r_{T}\right)\right|=v_{T i}\right)$. The horizontal lines correspond to the ion thermal velocities: bold solid line for $\mathrm{H}^{+}$ions and the dashed one for $\mathrm{O}_{2}^{+}$ ions.

$\Delta v(r)>v_{T \mathrm{i}}$, but on the other hand, the unstable threshold requires $\Delta v(r)<v_{\mathrm{i}} u_{\mathrm{da}} /\left(v_{\mathrm{dn}} p\right)$. For typical dusty plasma parameters in the planetary rings (Table 1$), v_{\mathrm{i}} u_{\mathrm{da}} /\left(v_{\mathrm{dn}} p\right) \geq 10^{8} \mathrm{~cm} \mathrm{~s}^{-1}$, and the instability condition from above is always satisfied. Therefore, the domain of unstable solutions in application to the planetary rings is only restricted from below by the condition $\Delta v(r)>v_{T i}$ (Fig. 4) and thus could be of importance for remote Saturn's rings (like E and $\mathrm{G}$ ).

Keeping in mind the ring conditions, where the dust can be polydisperse, and thus different dust components have different densities, it could be instructive to specify how our results are affected by the dust particle parameters, in particular the particle density $n_{\mathrm{d} 0}$ and the particle size $a$. Using the standard assumption $Z_{\mathrm{d}} \propto a$, the DAW frequency scales as $\omega \propto \omega_{\mathrm{pd}} \propto \sqrt{n_{\mathrm{d} 0} / a}$, while the growth rates of the wave instability (13)-(20) reveal slightly different dependencies: Eq. (13) results in $\mathfrak{J} \omega \propto n_{\mathrm{d} 0}$; Eqs. (15) and (20) give $\mathfrak{J} \omega \propto \sqrt{n_{\mathrm{d} 0} a}$; Eq. (19) leads to $\mathfrak{J} \omega \propto \sqrt{n_{\mathrm{d} 0}}$. Similarly, the conditions of the dust-acoustic instability, are also sensitive to the dust parameters. In particular, the onset of the instability (15) given by (16) yields $x_{\mathrm{cr}} \propto \sqrt{a^{-1} n_{\mathrm{d} 0}^{-1}}$. If we adopt the particle density as a power-law distribution $n_{\mathrm{d}}(a) \propto a^{-\mu}$ with $\mu \sim 4-6$ (relevant for dust distribution in remote planetary rings like $F$ and $G$, see Showalter et al. 1992; Showalter \& Cuzzi 1993) this immediately leads to the conclusion that the grains of small sizes are most likely to produce the DAW instability starting closest to the synchronous orbit.

\section{Summary}

We have studied the effect of dissipative forces on the propagation of the low-frequency dust-acoustic modes in the dusty plasma of planetary rings. It turned out that the collisional effects estimated for the region of Saturn's A and B rings may be large enough to affect the development of the low-frequency collective mode. In particular, we have demonstrated that the ion drag force related to the momentum transfer from the moving ions to the charged dust grains can be an important factor, which affects stability of the low-frequency perturbations in dusty plasma of the main planetary rings. Two features determine the exceptional role of the ion drag force - the occurrence of a ring atmosphere/ionosphere and of a relative motion between the plasma and dust particles. The relative velocity causes the existence of radial zones of transparency for DAW propagation and regions where dust-plasma waves are damped. We found that inside the co-rotation distance (where the plasma ions move slower than 
the dust grains) the ion drag force can be responsible for the excitation of dust-acoustic perturbations traveling along with dust flows. According to our model, the growth rate of this dissipative instability is strongly dependent on the radial distance from the planet. Reasonable estimations of the plasma parameters (Table 1) allow us to suggest that the boundary for the onset of the DAW instability located in the direct vicinity of the synchronize orbit. Note that the strong neutral gas damping (associated with dust-neutral momentum transfer) can quench the instability and move the boundary for the onset of the DAW instability inside the co-rotation distance (e.g. $x_{\mathrm{cr}, L}<x_{\mathrm{cr}, U}$ in Fig. 3). The source of free energy for this instability comes from the azimuthal drift of the dusty plasma components. Outside the co-rotation distance (where the plasma ions overtake the dust grains) the collisional process lead to damped perturbations, and thus the DAW can hardy be excited at radial distances corresponding to the main rings. The instability due to the ion drag force becomes possible again in the region where the relative ion velocities exceed their thermal speed. The latter could be of some importance e.g. for $E$ or $G$ rings, if the considered parameter regime is still valid in those remote regions.

Finally note that most of the plasma parameters used in the analysis fit approximately those estimated on the basis of the observations available from the few space missions flown up and made in the vicinity of the main A and B rings, while the quantities related to the dust grains (e.g. dust density, particle size and charge) correspond to plausible assumptions but might well have to be revised in the future. However, the presented theory allows us to clarify the role of the dust parameters. This is especially important for ring conditions because in reality the dust in the planetary rings is polydisperse and comes in almost continuous range from macromolecules to rock fragments. According to our predictions, the smaller the charged particles are, the larger the growth rate of the DAW instability and the smaller the distance from the synchronous orbit for the onset of the instability. It is possible that due to these characteristics the discovered instability could be of some importance for the formation and evolution Saturnian "spokes" occurring in the vicinity of the synchronous orbit. This issue deserved further examination, but is outside the scope of the presented investigation.

Acknowledgements. F.V. thanks the Fonds voor Wetenschappelijk Onderzoek (Vlaanderen) for a research grant. The critical remarks by the referee are gratefully acknowledged.

\section{References}

Barkan, A., Merlino, R. L., \& D’Angelo N. 1995, Phys. Plasmas, 2, 3563 Barnes, M. S., Keller, J. H., Forster, J. C., et al. 1992, Phys. Rev. Lett., 68, 313 Bliokh, P. V., \& Yaroshenko, V. V. 1985, Sov. Astron., Engl. Transl., 29, 330

Bliokh, P. V., Sinitsyn, V. G., \& Yaroshenko, V. V. 1995, Dusty and SelfGravitational Plasmas in Space (Kluwer, Dordrecht)

Chu, J. H., Ji-Bin Du, \& Lin, I. 1994, J. Phys. D: Appl. Phys., 27, 296

Cramer, N., Verheest, F., Cattaert, T., et al. 2004, Phys. Plasmas, 11, 4589 D’Angelo, N. 1995, J. Phys. D: Appl. Phys. 28, 1009

D’Angelo, N., \& Merlino, R. L. 1996, Planet. Space Sci. 44, 1593

Daugherty, J. E., \& Graves, D. B. 1995, J. Appl. Phys., 78, 2279

Epstein, P. S. 1921, Phys. Rev., 23, 710

Fortov, V. E., Khrapak, A. G., Khrapak, S. A., et al. 2000, Phys. Plasmas, 7, 1374

Fortov, V. E., Usachev, A. D., \& Zobnin, A. V. 2003, Phys. Plasmas, 10, 1199

Gurnett, D. A., Kurth, W. S., Hospodarsky, G. B., et al. 2005, Science, 307, 1255 Joyce, G., Lampe, M., \& Ganguli, G. 2002, Phys. Rev. Lett. 88, 095006 Ip, W.- H. 1995, Icarus, 115, 295

Itikawa, Y., Ichimura, A., Onda, K., et al. 1989, J. Phys. Chem., 18, 23

Ivlev, A. V., \& Morfill, G. E. 2000, Phys. Plasmas, 7, 1094

Ivlev, A. V., Samsonov, D., Goree, J., et al. 1999, Phys. Plasmas, 6, 741

Li, F., \& Havnes, O. 2000, Planet. Space Sci., 48, 117

Khrapak, S., Samsonov, D., Morfill, G. E., et al. 2003, Phys. Plasmas, 10, 1

Khrapak, S., \& Yaroshenko, V. V. 2003, Phys. Plasmas, 10, 4616

Khrapak, S. A., Ivlev, A. V., Morfill, G. E., \& Thomas, H. M. 2002, Phys. Rev. E, 66, 046414

Kilgore, M. D., Daugherty, D. E., Porteus, R. K., et al. 1993, J. Appl. Phys., 73, 7195

Liu, B., Goree, J., Nosenko, V., \& Boufendi, L. 2003, Phys. Plasmas, 10, 9

Melandsø, F., Aslaksen, T. K., \& Havnes, O. 1993, J. Geophys. Res. 98, 13315

Mendis D. A. 2005, dust-plasma interactions in space, in New Vistas in Dusty Plasmas: 4th Int. Conf. Physics of Dusty Plasmas, ed. L. Boufendi, M. Mikkikian, \& P. K. Shukla (Melville, NY: AIP), 799, 583

Mikailovskii, A. B. 1973, Theory of plasma instabilities. Vol. 1: Instabilities of homogeneous plasmas (New York, London: Consultants Bureau)

Pieper, J. B., \& Goree, J. 1996, Phys. Rev. Lett., 77, 3137

Rao, N. N., Shukla, P. K., \& Yu, M. Y. 1990, Planet. Space Sci. 38, 543

Rosenberg, M. 1993, Planet. Space Sci., 41, 229

Rosenberg, M. 1996, J. Vac. Sci. Technol. A, 14, 631

Rosenberg, M. 2002, J. Plasma Phys., 67, 235

Showalter, M. R., Pollack, M. E., Oskert, M. E., et al. 1992, Icarus, 100, 394

Showalter, M. R., \& Cuzzi, J. N. 1993, Icarus, 103, 124

Smith, G. J., Gao, R. S., Lindsay, B. G., et al. 1996, Phys. Rev. A, 53, 1581

Thompson, C., Barkan, A., D’Angelo, N., \& Merlino, R. L. 1997, Phys. Plasmas, 4,2331

Verheest, F. 2000, Waves in Dusty Space Plasmas (Dordrecht: Kluwer)

Waiter, Jr., J. H., Cravens, T. E., Ip, W.-H., et al. 2005, Science, 307, 1260

Winske, D., Gary, S. P., Jones, M. E., et al. 1995, Geophys. Res. Lett., 22, 2069

Yaroshenko, V. V., Anaratone, B. M., Khrapak, S., et al. 2004, Phys. Rev. E, 69, 066401

Young, D. T., Berthelier, J.-J., Blanc, M., et al. 2005, Science, 307, 1262 